\title{
MENINGOENCEFALITE GRANULOMATOSA EM CÃO
}

\author{
GRANULOMATOUS MENINGOENCEPHALITIS IN A DOG
}

Enio Pedone BANDARRA'; Renée LAUFER²; Júlio Lopes SEQUEIRA³ ${ }^{3}$ Ragnar Franco SCHAMALL²; Laura Maria Alvarez de FIGUEIREDO'; Márcio Botelho de CASTRO²

\begin{abstract}
RESUMO
A Meningoencefalite Granulomatosa (MEG)é um processo inflamatório não supurativo do Sistema Nervoso Central(SNC). A MEG tem sido descrita desde 1972, e uma grande variedade de termos já foi usada para designar tal doença (THOMAS; EGER ${ }^{14}, 1989$ ). Este trabalho tem o objetivo de relatar o caso de um cão, fêmea, da raça Dachshund, com 3 anos e 8 meses de idade, que foi encaminhado ao Hospital Veterinário da Faculdade de Medicina Veterinária e Zootecnia, UNESP, Botucatu, com quadro de incoordenação havia 10 dias, evoluindo para tremores e desvio lateral da cabeça. Após exame clínico e constatando-se a irreversibilidade do processo, o animal foi submetido à eutanásia. A necrópsia revelou extensa área de leucomalácia, e os estudos histopatológicos mostraram tratarse de MEG, doença de extrema controvérsia e rara ocorrência na literatura compulsada.
\end{abstract}

UNITERMOS: Meningoencefalite; Granuloma; Sistema Nervoso Central; Cães; Leucemia

\section{INTRODUÇÃO}

A Meningoencefalite Granulomatosa (MEG) é uma doença inflamatória, não supurativa do Sistema Nervoso Central (SNC), descrita em várias espécies animais, incluindo caninos, felinos, eqüinos e bovinos ${ }^{2,9}$. Foi reconhecida inicialmente em 1972 por FANKHAUSER et al. ${ }^{6}$ e, desde então, tem sido descrita nos Estados Unidos, na Nova Zelândia e na Austrália.

Durante determinado periodo, aquela doença era considerada de caráter neoplásico, sendo chamada de Reticulose Primária do $\mathrm{SNC}^{16}$. Em estudos mais recentes, já se reconhece o aspecto inflamatório, caracterizado pelo aumento do número de histiócitos, linfócitos, monócitos e plasmócitos, agrupados em manguitos perivasculares, formando granulomas ${ }^{14}$.

As lesões da MEG podem ser disseminadas, focais ou de manifestação ocular. A forma disseminada já foi denominada Reticulose Inflamatória e Encefalite Histiocitária e a forma focal, Reticulose Neoplásica ${ }^{11}$.

Não há concordância na literatura consultada quanto ao sexo e raça mais afetados, bem como a faixa etária acometida, podendo variar de 8 meses a 8 anos de ida$\mathrm{de}^{5,14,15}$

\section{MATERIAL E MÉTODO}

Um cão fềmea, com três anos e oito meses de idade, da raça Dachshund, foi trazido à Clínica de Pequenos Animais da Faculdade de Medicina Veterinária e Zootecnia, UNESP, Botucatu, com histórico de incoordenação motora havia dez dias, agravada significantemente nos últimos cinco dias.

O animal foi submetido a exame clínico e neurológico completo, além de exames complementares (hemograma e análise do líquido cefalorraquidiano - LCR).

O quadro evoluiu por dois meses, sendo, após esse período, indicado o sacrifício do animal, devido à irreversibilidade do processo.

O animal foi encaminhado ao Serviço de Patologia, onde foi realizado exame necroscópico, segundo técnicas preestabelecidas, colhendo-se para histopatologia fragmentos de cérebro, figado e rins, que foram fixados em solução tamponada de formol a $10 \%$ e incluídos em blocos de parafina. O material foi submetido a cortes entre 5 e 7 . micrômetros, sendo corados pelos métodos de Hematoxilina$\operatorname{Eosina}^{10}$. Posteriormente, no intuito de se estabelecer uma etiologia e diagnósticos diferenciais, foram feitas colorações de Gram, PAS (Periodic Acid-Schiff) e Woelcke para bainha de mielina ${ }^{10}$.

\footnotetext{
I - Professor Assistente Doutor - Faculdade de Medicina Veterinária e Zootecnia da UNESP-Botucatu, SP

2 - Médico Veterinário Residente - Faculdade de Medicina Veterinária e Zootecnia da UNESP-Botucatu, SP

3 - Professor Assistente - Faculdade de Medicina Veterinária e Zootecnia da UNESP- Botucatu, SP
} 
BANDARRA, E.P.; LAUFER, R.; SEQUEIRA, J.L.; SCHAMALL, R.F.; FIGUEIREDO, L.M.A.; CASTRO, M.B. Meningoencefalite granulomatosa em cão. Braz. J. vet. Res. anim. Sci., São Paulo, v.32, n.2, p.101-4. 1995.

\section{RESULTADOS}

Durante a avaliação clínica o animal apresentou os parâmetros fisiológicos dentro dos valores normais para a espécie. As anormalidades reveladas pelo exame físico se limitavam ao $\mathrm{SNC}$, representadas por tremores, desvio lateral da cabeça para a esquerda, desequilíbrio, tendência para rotação em círculos amplos, no sentido anti-horário, nistagmo rotatório e vertical, além de hipermetria.

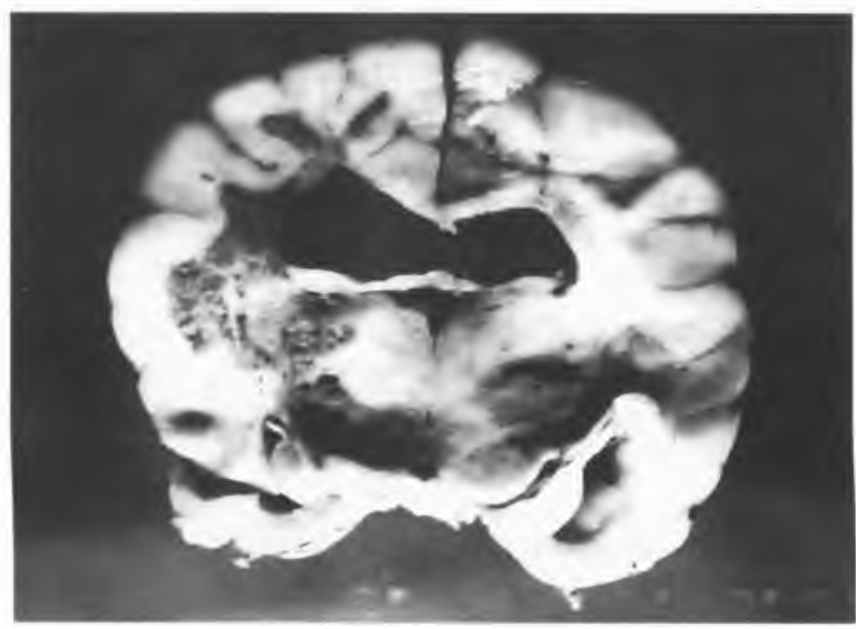

FIGURA 1

Cérebro. Extensa área de leucomalácia no hemisfério cerebral esquerdo.

O hemograma estava normal e a análise do LCR demonstrou aumento da proteína liquórica $(543,9 \mathrm{mg} / \mathrm{dl})$ e teste de Pandy com três cruzes. Na citologia liquórica notou-se pleocitose ( $130 \mathrm{WBC} / \mathrm{ml}$ ) com predomínio de linfócitos e monócitos, alguns macrófagos íntegros com atividade fagocitária e raros eosinófilos.

O exame necroscópico revelou intenso edema cerebral, acompanhado de congestão dos vasos meningeanos do hemisfério esquerdo. Ao corte transversal do cérebro em fatias de aproximadamente $1 \mathrm{~cm}$ de espessura, observou-se extensa área de malácia na substância branca do hemisfério esquerdo, circundando o córtex cerebral e o ventrículo lateral. No tálamo também havia áreas de necrose (Fig. 1). Os demais achados macroscópicos foram: hidropericárdio, gastroenterite catarral e moderada infecção por Dipilydium caninum.

Na análise histopatológica da substância branca, observaram-se agregados densos de células mesenquimais, em organização perivascular, formando granulomas. Havia macrófagos, alguns fagocitando detritos celulares do tecido nervoso necrosado, linfócitos, plasmócitos e, em menor número, células gigantes e neutrófilos (Fig. 2).

Alguns granulomas se uniam, com necrose do tecido adjacente. Notou-se intensa necrose de liquefação, edema e gliose

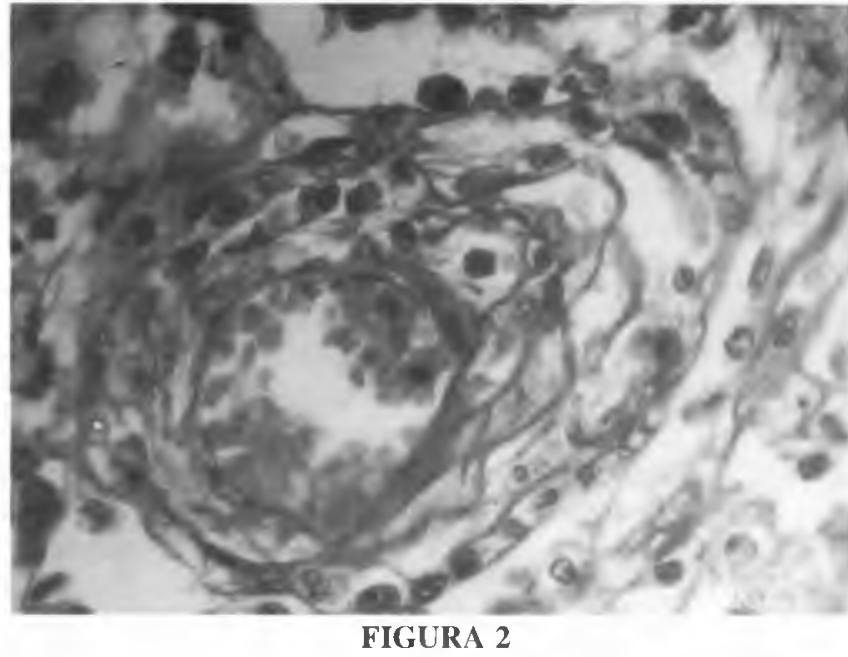

Substância branca cerebral. Granuloma composto por macrófagos e plasmócitos. PAS, 40x.

moderada. Os manguitos perivasculares eram compostos basicamente por linfócitos e monócitos, com raros polimorfonucleares. Alguns vasos apresentavam esclerose de suas paredes (Fig. 3).

A substância cinzenta mantinha seu aspecto normal.

O cerebelo apresentava baixa celularidade da camada granular e diminuição do número de células de Purkinje (Fig. 4)

As colorações especiais (PAS e Gram) não demonstraram presença de bactérias ou fungos. Com o método de Woelcke ressaltou-se a intensa desmielinização da substância branca encefálica e cerebelar.

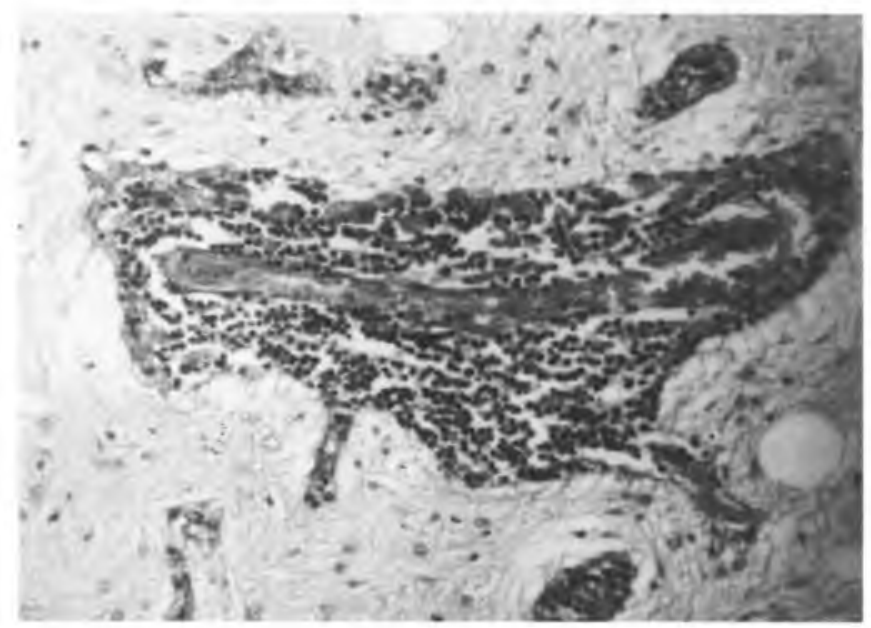

FIGURA 3

Substância branca cerebral. Manguito perivascular mononuclear. PAS, 25x. 


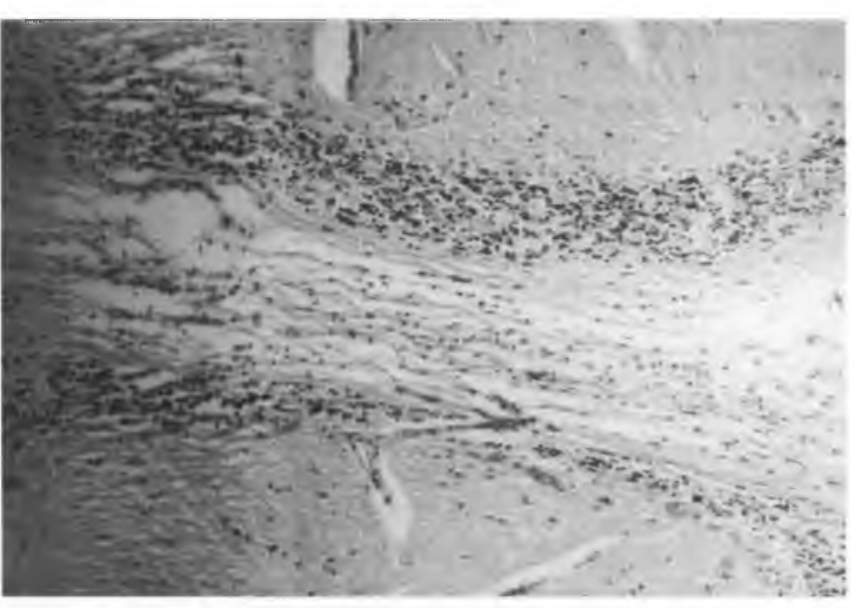

FIGURA 4

Cerebelo. Baixa celularidade da camada granular, com diminuição do número de células de Purkinje e desmielinização da substância branca cerebelar. Coloração de Woelcke, 12,5x.

\section{DISCUSSÃO E CONCLUSÃO}

Devido ao envolvimento predominantemente vascular, o caráter reticuloproliferativo das lesões e a predileção pela substância branca cerebral, foi feito o diagnóstico de Meningoencefalite Granulomatosa ${ }^{3,8,9}$.

Os sinais clínicos observados estavam relacionados com a localização anatômica das lesões, o que já foi descrito por outros autores $^{2,3,8,12}$, bem como seu aparecimento agudo e evolução lenta ${ }^{3,8}$.

Devido a estas características, os sinais clínicos da MEG lembram os de outras entidades neurológicas, sugerindo-se que, apesar da pequena freqüência de ocorrência da MEG canina, esta deve ser considerada como diagnóstico diferencial de uma variedade de doenças do $\mathrm{SNC}^{12}$.

Nas encefalites bacterianas há dor e rigidez cervical, além de febre $^{2}$. Na análise do líquor observa-se concentração de proteína aumentada (100 a $1.000 \mathrm{mg} / \mathrm{dl})$ e pleocitose polimorfonuclear (100 a 1.000 cels $/ \mathrm{mm}^{3}$ ). Microscopicamente nota-se uma reação inflamatória supurativa. Na MEG foi observada febre no curso agudo da doença ${ }^{11,13}$, porém, os demais achados diferem em relação ao tipo de infiltrado celular liquórico e achados microscópicos.

Já nos casos de toxoplasmose e encefalites micóticas (criptococose, blastomicose e histoplasmose) há sinais de envolvimento sistêmico, com sorologia positiva, e no líquor observa-se pleocitose mono e polimorfonuclear $\left(40 \text { a } 100 \mathrm{cels} / \mathrm{mm}^{3}\right)^{3}$.

Cães com cinomose apresentam sorologia positiva à virose $\mathrm{e}$ presença de anticorpos fluorescentes em esfregaços de conjuntiva. Acometendo com mais freqüência animais jovens, com menos de um ano de idade. Nota-se no LCR pleocitose mononuclear moderada $\left(8 \text { a } 40 \text { cels } / \mathrm{mm}^{3}\right)^{2}$, além de, histopatologicamente se observar distribuição topográfica das lesões diferente da da MEG e presença de corpúsculos de inclusão de Sinegaglia-Leintz ${ }^{3}$.

Os outros diferenciais da forma focal da MEG incluem tumores primários ou metastáticos do $\mathrm{SNC}$, onde o líquor se apresenta normal e o diagnóstico "post mortem" é essencial ${ }^{2}$.

A análise do LCR é o meio mais útil no auxílio de se estabelecer um diagnóstico de $\mathrm{MEG}^{2}, 3,7,11,13$, sendo característico um aumento da proteína $(40$ a $100 \mathrm{mg} / \mathrm{dl})$ e uma pleocitose $\left(50\right.$ a $\left.600 \mathrm{WBC} / \mathrm{mm}^{3}\right)$, principalmente mononuclear (linfóticos e monócitos) ${ }^{2}$.

A hiperproteinorraquia pode ser resultado da produção local de IgG, e não de alterações na barreira hematoencefálica'".

Um outro método que pode ser utilizado no diagnóstico clínico da MEG é a corticoterapia, em que se nota a melhora dos sinais clínicos, bem como a diminuição da celularidade do líquor ${ }^{12}$.

O diagnóstico definitivo geralmente requer o estudo histopatológico do $\mathrm{SNC}^{11}$.

A etiologia desse processo não é conhecida ${ }^{1,2,4,14,15}$. Nenhum agente que ocasionalmente causaria infecção granulomatosa, como o da tuberculose, os da micose ou os da toxoplasmose, foi identificado.

Vários autores acreditam ser uma resposta alterada ao vírus da cinomose ou a sua forma de "encefalite do cão idoso" 1, 2, 14, 15, 16. VANDEVELDE et al. ${ }^{16}$ (1978) reconheceu a presença do vírus da cinomose em um caso, por técnicas de imunofluorescência com anticorpos antivírus ${ }^{16}$. Já em outro trabalho mais recente, 21 casos de cães com $\mathrm{MEG}$ foram submetidos a técnicas de imuno-histoquímica, para comprovar a presença do antígeno do vírus da cinomose, tendo sido obtidos resultados negativos, sugerindo a não participação deste agente nesse processo ${ }^{14}$.

Na literatura nacional indexada não se encontram descrições dessa doença, o que pode ser explicado não só pela dificuldade de diagnóstico clínico preciso, como também pela menor freqüência de exame do SNC nas necrópsias.

No presente caso, o eventual agente etiológico também não foi identificado, ficando clara a necessidade de se fazer rotineiramente explorações clínicas, laboratoriais e anatomopatológicas do SNC e, ainda, o emprego de técnicas mais sofisticadas (tais como imuno-histoquímica, microscopia eletrônica, imunofluorescência) no auxílio ao diagnóstico. 


\begin{abstract}
SUMMARY
Granulomatous Meningoencephalitis (GME) is an inflamatory, non suppurative disease of the Central Nervous System. This disease has been described since 1972 and a great variety of terms have been used to name (THOMAS; EGGER ${ }^{14}, 1989$ ). This paper describes a case of a 3 years and 8 months old, female Dachshund, that was brought to the Veterinary Teaching Hospital at UNESP, Botucatu, São Paulo, Brazil, showing incoordenation during the last 10 days and head tilt. After careful examination, concluding to irreversibility of the process, the dog was euthanatised. Necropsy findings were necrosis of the white cerebral matter and in histopathologic examination of the CNS was diagnosticated GME.
\end{abstract}

UNITERMS: Meningoencephalitis; Granuloma; Central Nervous System; Dogs; Leukaemia

\title{
REFERÊNCIAS BIBLIOGRÁFICAS
}

1-ALLEY, M.R.; JONES, B.R.; JOHNSTONE, A.C. Granulomatous meningoencephalomyelitis of dogs in New Zealand. New Zealand Veterinary Journal, v.31, p.117-9, 1983.

2-BRAUND, K.G. Granulomatous meningoencephalomyelitis. Journal of the American Veterinary Medical Association, v.186, p.138-41, 1985.

3-BRAUND, K.G.; VANDEVELDE, M.; WALKER, T.L.; REDDING, R.W.Granulomatous meningoence-phalomyelitis in six dogs. Journal of the American Veterinary Medical Association, v.172, p.1195-200, 1978.

4-CORDY, D.R. Canine granulomatous meningoencephalomyelitis. Veterinary Pathology, v.16, p.325-33, 1979.

5-ETTINGER, S.J.Textbook of veterinary internal medicine disease of the dog and cat. 3.ed. Philadelphia, W.B. Saunders, 1989.

6-FANKHAUSER, R.; FATZER, R.; LUGINBÜHL, H., 1972. apud THOMAS; EGER ${ }^{14}, 1989$. p.289.

7-GARMER, N.L.; NAESER, P.; BERGMAN, A.J. Reticulosis of the eyes and central nervous system in a dog. Journal of Small Animal Practice, v.22, p.39-45, 1981.

8-GLASTONBURY, J.R.W.; FRAUENFELDER, A.R. Granulomatous meningoencephalomyelitis in a dog. Australian Veterinary Journal, v.57, p. 186-8, 1981.

9-JUBB, K.V.F.; KENNEDY, P.C.; PALMER, N.Pathology of domestical animals s. California, Academic Press, 1993. v.1, Chap 3: The nervous system.

10-LUNA, L.G. Manual of histologic staining methods of the Armed Forces Institute of Pathology. New York, McGrawHill, 1968.
11-MURTAUGH, R.J.; FENNER, W.R.; JOHNSON, G.C. Focal granulomatous meningoencephalomyelitis in a pup. Journal of the American Veterinary Medical Association, v.187, p.835-6, 1985.

12-RUSSO, M.E. Primary reticulosis of the central nervous system in dogs.Journal of the American Veterinary Medical Association, v.174, p.492-500, 1979.

13-SORJONEN, D.C. Clinical and histophatological features of granulomatous meningoencephalomyelitis in dogs. Journal of the American Animal Hospital Association, v.26, p.141-7, 1990.

14-THOMAS, J.B.; EGER, C. Granulomatous meningoencephalomyelitis in 21 dogs. Journal of Small Animal Practice, v.30, p.287-93, 1989.

15-VANDEVELDE, M. Primary reticulosis of the central nervous system.Veterinary Clinics of North America. Small Animal Practice, v.10, p.57-63, 1980.

16 -VANDEVELDE, M.; KRISTENSEN, B.; GREENE, C.E. Primary reticulosis of the central nervous system in the dog. Veterinary Pathology, v.15, p.673-5, 1978.
Recebido para publicação em 04/04/94 Aprovado para publicação em 07/11/94 